ALEA, Lat. Am. J. Probab. Math. Stat. 16, 1129-1140 (2019)

DOI: $10.30757 /$ ALEA.v16-42

\title{
Exponent for sets of frequently visited points of a simple random walk in two dimensions
}

\section{Izumi Okada}

Motooka 744, Nishi-ku, Fukuoka 819-0395, Japan

E-mail address: iokada@math.kyushu-u.ac.jp

\begin{abstract}
Herein, we present a study on frequently visited sets in a simple random walk in $\mathbb{Z}^{2}$. We estimate the expectation of numbers of $j$-tuples of favorite points and obtain an exact exponent.
\end{abstract}

\section{Introduction}

We study properties of special points, called favorite points in a random walk range in $\mathbb{Z}^{2}$, which are sites at which the local time of a simple random walk is close to the most frequently visited site. In particular, we observe the number of sets of favorite points in $\mathbb{Z}^{2}$. We call these sets favorite sets. We will define the favorite set $\mathcal{R}_{j, n}(\alpha)$ and obtain certain asymptotic estimates of

$$
\mid\left\{\vec{x} \in \mathcal{R}_{j, n}(\alpha): d\left(x_{i}, x_{l}\right) \leq n^{\beta} \text { for any } 1 \leq i, l \leq j\right\} \mid
$$

for $\vec{x}=\left(x_{1}, \ldots, x_{j}\right), 0<\alpha, \beta<1$ and $j \in \mathbb{N}$.

Our motivation behind this study is to understand the relationship between the special points of a random walk and that of the Gaussian free field, such as late points and high points. In fact, there are several known related results. Dembo et al. (2006) and Brummelhuis and Hilhorst (1991) computed the number of pairs of late points in two dimensions. Daviaud (2006) estimated that the high points of the Gaussian free field in two dimensions has the same forms as that in Brummelhuis and Hilhorst (1991); Dembo et al. (2006). We obtain the same form for favorite points in Okada $(2019+)$. In each case, there is a certain consistency of the exponents.

To state our main result, we introduce the following notations. Let $d$ be the Euclidean distance and $\mathbb{N}:=\{1,2, \cdots\}$. For $n \in \mathbb{N}$, let $D(x, r):=\left\{y \in \mathbb{Z}^{2}\right.$ : $d(x, y)<r\}$ and for any $G \subset \mathbb{Z}^{2}, \partial G:=\left\{y \in G^{c}: d(x, y)=1\right.$ for some $\left.x \in G\right\}$. For $x \in \mathbb{Z}^{2}$, we sometimes omit \{\} while writing a one-point set $\{x\}$. Let $\left(S_{k}\right)_{k=0}^{\infty}$ be a simple random walk on the 2 -dimensional square lattice. Let $P^{x}$ denote the

Received by the editors May 4th, 2018; accepted October 9th, 2019.

2010 Mathematics Subject Classification. 82C44, 60K35, 60G70.

Key words and phrases. Simple random walks, local times. 
probability of the simple random walk starting at $x$. Then, we simply write $P$ for $P^{0}$. For any $A \subset \mathbb{Z}^{2}$, let $K(n, A)$ be the number of times the simple random walk visits $A$ up to time $n$, that is, $K(n, A)=\sum_{i=0}^{n} 1_{\left\{S_{i} \in A\right\}}$. For $x \in \mathbb{Z}^{2}$, let $T_{x}:=\inf \left\{m \geq 1: S_{m}=x\right\}$ and $\tau_{n}:=\inf \left\{m \geq 0: S_{m} \in \partial D(0, n)\right\}$.

In two dimensions, favorite points of simple random walks have been studied. Originally, Erdös and Révész (1987) suggested several problems related to the local time of the simple random walk. Approximately forty years later, Dembo, Peres, Rosen and Zeitouni (Dembo et al., 2007, 2001, 2004) solved many open problems regarding the simple random walk in $\mathbb{Z}^{2}$. Dembo et al. (2001) showed that for the simple random walk in $\mathbb{Z}^{2}$

$$
\lim _{n \rightarrow \infty} \frac{\max _{x \in \mathbb{Z}^{2}} K(n, x)}{(\log n)^{2}}=\frac{1}{\pi} \quad \text { a.s. }
$$

It can easily yield the following

$$
\lim _{n \rightarrow \infty} \frac{\max _{x \in \mathbb{Z}^{2}} K\left(\tau_{n}, x\right)}{(\log n)^{2}}=\frac{4}{\pi} \quad \text { a.s. }
$$

given that $\log \tau_{n} / \log n^{2} \rightarrow 1$ almost surely as $n \rightarrow \infty$. In Okada $(2019+)$, we set $x$ to be $\alpha$-favorite point for $0<\alpha<1$ if $K\left(\tau_{n}, x\right)$ is larger than $4 \alpha(\log n)^{2} / \pi$. In Dembo $(2005,2006)$; Dembo et al. (2004, 2006), the authors suggested the open problem for structures of $\alpha$-favorite points. Next, for $0<\alpha<1$, we define $\alpha$-favorite sets in $\mathbb{Z}^{2}$ such that

$$
\mathcal{R}_{j, n}(\alpha):=\left\{\vec{x} \in D(0, n)^{j}: K\left(\tau_{n},\left\{x_{1}, \ldots, x_{j}\right\}\right) \geq\left\lceil\frac{4 \alpha j}{\pi}(\log n)^{2}\right\rceil\right\},
$$

where $\lceil a\rceil$ denotes the smallest integer $n$ with $n \geq a$.

Now, we provide our main result on the structures of favorite sets.

Theorem 1.1. For any $0<\alpha, \beta<1$

$$
\lim _{n \rightarrow \infty} \frac{\log E\left[\mid\left\{\vec{x} \in \mathcal{R}_{j, n}(\alpha): d\left(x_{i}, x_{l}\right) \leq n^{\beta} \text { for any } 1 \leq i, l \leq j\right\} \mid\right]}{\log n}=\hat{\rho}_{j}(\alpha, \beta),
$$

where

$$
\hat{\rho}_{j}(\alpha, \beta):= \begin{cases}2+2(j-1) \beta-\frac{2 j \alpha}{(1-\beta)(j-1)+1} & \left(\beta \leq 1+\frac{1-\sqrt{j \alpha}}{j-1}\right), \\ 2(j+1-2 \sqrt{j \alpha}) & \left(\beta \geq 1+\frac{1-\sqrt{j \alpha}}{j-1}\right) .\end{cases}
$$

The above exponent is the same as that of the main result in Okada (2019).

Remark 1.2. We expect the following: the limit of $\log \mid\left\{\vec{x} \in \mathcal{R}_{j, n}(\alpha): d\left(x_{i}, x_{l}\right) \leq\right.$ $n^{\beta}$ for any $\left.1 \leq i, l \leq j\right\} \mid / \log n$ as $n \rightarrow \infty$ exists almost surely. However, we expect that this proof does not contribute to this study. Hence, we have omitted the result.

Now, we explain the basic approach of this paper. To show Theorem 1.1, we find an appropriate estimate of

$$
\begin{aligned}
& \sum_{\substack{d\left(x_{i}, x_{l}\right) \leq n^{\beta}, x_{i} \in \mathbb{Z}^{2}, 1 \leq \forall i, l \leq j}} P\left(\vec{x} \in \mathcal{R}_{j, n}(\alpha)\right) . \\
&\left.\left.\mathcal{R}_{j, n}(\alpha): d\left(x_{i}, x_{l}\right) \leq n^{\beta} \text { for any } 1 \leq i, l \leq j\right\} \mid\right] \\
&
\end{aligned}
$$

Note that the position of a $j$-tuple of points determines the value of $P\left(\vec{x} \in \mathcal{R}_{j, n}(\alpha)\right)$. This value can be expressed by a matrix constructed from $G_{n}(x, y):=$ 
$\sum_{m=0}^{\infty} P^{x}\left(S_{m}=y, m<\tau_{n}\right)$ for $x, y \in D(0, n)$, which is a Green's function of the walk killed when it exits $D(0, n)$. We shall show that uniformity is achieved in $x_{1}, \ldots, x_{j} \in D(0, n / 10)$,

$$
P\left(\vec{x} \in \mathcal{R}_{j, n}(\alpha)\right) \approx \exp \left(-2 \alpha j \lambda\left(\left(\frac{\pi G_{n}\left(x_{i}, x_{l}\right)}{2 \log n}\right)_{1 \leq i, l \leq j}\right)^{-1} \log n\right),
$$

where $a_{n} \approx b_{n}$ means $\log a_{n} / \log b_{n} \rightarrow 1$ as $n \rightarrow \infty$ for any sequence and $\lambda(A)$ is the maximal eigenvalue of $A$. We obtain (1.1) using the same argument as that of Csáki et al. (2005) in Lemma 2.9. After computing (1.1), we sum it using the similarity between Green's functions and ultrametric matrices (see Section 2.2) as well as Okada (2019). As a different point from the proof of the result in Okada (2019), we deal with the maximal eigenvalues of ultrameric matrices while we compute the summation over all the entries of inverse matrices of ultrameric matrices in Okada (2019). Then, the key estimate in this paper corresponds to Proposition 2.2. Essentially, we need original computations.

\section{Proofs}

In this section, we provide the proof for Theorem 1.1. In Sections 2.1 and 2.2, we prepare estimates to show Theorem 1.1 and show it in Section 2.3. Hereafter, the values of the constants $c$ and $C$ may vary from place to place.

2.1. Green's function. In this section, we introduce estimates of a Green's function for a simple random walk in $\mathbb{Z}^{2}$. From Exercise 1.6.8 of Lawler (1991) or (4.1) and (4.3) in Rosen (2005), we have that, uniformly in $0<|x|<R$,

$$
P^{x}\left(T_{0}<\tau_{R}\right)=\frac{\log (R /|x|)+O\left(|x|^{-1}\right)}{\log R}\left(1+O\left((\log |x|)^{-1}\right)\right) .
$$

Recall that for $x, y \in D(0, n)$, we set $G_{n}(x, y)=\sum_{m=0}^{\infty} P^{x}\left(S_{m}=y, m<\tau_{n}\right)$. In addition, by Proposition 1.6.7 in Lawler (1991) or (2.1) in Rosen (2005), we have that for any $x \in D(0, n)$

$G_{n}(x, 0)=\sum_{m=0}^{\infty} P^{x}\left(S_{m}=0, m<\tau_{n}\right)=\frac{2}{\pi} \log \left(\frac{n}{d(x, 0)^{+}}\right)+O\left(\left(d(x, 0)^{+}\right)^{-1}+n^{-1}\right)$,

$G_{n}(0,0)=\frac{2}{\pi} \log n+O(1)$

where $a^{+}:=a \vee 1$. Therefore, we have that for $x, y \in D(0, n / 3)$

$$
G_{n}(x, y)=\frac{2}{\pi} \log \left(\frac{n}{d(x, y)^{+}}\right)+O(1)
$$

since

$$
\begin{aligned}
& G_{n}(x, y) \leq \sum_{m=0}^{\infty} P^{x-y}\left(S_{m}=0, m<\tau_{4 n / 3}\right)=\frac{2}{\pi} \log \left(\frac{n}{d(x, y)^{+}}\right)+O(1) \\
& G_{n}(x, y) \geq \sum_{m=0}^{\infty} P^{x-y}\left(S_{m}=0, m<\tau_{2 n / 3}\right)=\frac{2}{\pi} \log \left(\frac{n}{d(x, y)^{+}}\right)+O(1)
\end{aligned}
$$


2.2. Matrix argument. In this section, we provide matrix properties for ultrametric matrices. We introduce the following notations and introduce the results proved by Okada (2019) et al. Our main aim in this section is to prove Proposition 2.4.

Fix $0<\alpha, \beta<1$ and $j \in \mathbb{N}$. Given $0<\eta<(1-\beta) \wedge \beta$, let $\mathcal{M}_{j}^{\beta, \eta}$ be a subset of $j \times j$-(symmetric) strictly ultrametric matrices $\left(a_{i, l}\right)_{1 \leq i, l \leq j}$ satisfying $a_{i, i}=1$, $1-\beta \leq a_{i, l} \leq 1-\eta$ for $1 \leq i \neq l \leq j$ (see the explanation of ultrametric matrices in Dellacherie et al., 2014; Martínez et al., 1994). Hereafter, we denote $\left(a_{i, l}\right)_{1 \leq i, l \leq j}$ as $A$. For $j_{k} \in \mathbb{N}$, let $A_{k}:=\left(a_{i, l}^{(k)}\right)_{1 \leq i, l \leq j_{k}} \in \mathcal{M}_{j_{k}}^{\beta, \eta}(\forall k=1, \ldots, m)$ and $j=$ $\sum_{k=1}^{m} j_{k}$. For the injective function $\sigma_{k}:\left\{1, \ldots, j_{k}\right\} \rightarrow\{1, \ldots, j\}(\forall k=1, \ldots, m)$ with $\cup_{k=1}^{m} \operatorname{Im} \sigma_{k}=\{1, \ldots, j\}$ and $s \leq \min \left\{a_{i, l}^{(k)} \mid k \in\{1, \ldots, m\}, i, l \in\left\{1, \ldots, j_{k}\right\}\right\}$, we let $A=A_{1}^{\sigma_{1}} \boxplus_{s} \ldots \boxplus_{s} A_{m}^{\sigma_{m}}$ if

$$
a_{i, l}:= \begin{cases}a_{\sigma_{k}^{-1}(i), \sigma_{k}^{-1}(l)}^{(k)} & \text { for } i, l \in \operatorname{Im} \sigma_{k}, \quad k=1, \ldots, m, \\ s & \text { otherwise. }\end{cases}
$$

Proposition 3.4 in Dellacherie et al. (2014) or Proposition 5.1 in Okada (2019) yields the following:

Proposition 2.1. It holds that for $j \geq 2$ with $j \in \mathbb{N}, A \in \mathcal{M}_{j}^{\beta, \eta}$ satisfies the following: there exist $A_{k} \in \mathcal{M}_{j_{k}}^{\beta, \eta}$ and $\sigma_{k}$ for $k=1, \ldots, m$ with $m \geq 2$ such that $A=A_{1}^{\sigma_{1}} \boxplus_{s} \ldots \boxplus_{s} A_{m}^{\sigma_{m}}$, where $s<\min \left\{a_{i, l}^{(k)} \mid k \in\{1, \ldots, m\}, i, l \in\left\{1, \ldots, j_{k}\right\}\right\}$.

To state the following proposition, for a regular matrix $A$, let $y_{1}(A), \ldots, y_{j}(A)$ be the solution that satisfies $A\left(y_{1}(A), \ldots, y_{j}(A)\right)^{T}=\overrightarrow{1}^{T}$, where $\overrightarrow{1}=(1, \ldots, 1)$ and $\chi(A):=\sum_{i=1}^{j} y_{i}(A)$ if $\chi(A)$ is well defined. Note that if $A$ is a regular matrix, $\chi(A)$ corresponds to the summation over all the entries of $A^{-1}$. Theorem 3.5 in Dellacherie et al. (2014) yields that a matrix included in $\mathcal{M}_{j}^{\beta, \eta}$ is a regular matrix. In Okada (2019), we defined $\Xi$ inductively as follows: for $A \in \mathcal{M}_{j}$ which is equal to $A_{1}^{\sigma_{1}} \boxplus_{s} \ldots \boxplus_{s} A_{m}^{\sigma_{m}}$, set

$$
\Xi(A):=\sum_{k=1}^{m} \Xi\left(A_{k}\right)+(m-1)(1-s),
$$

where $\Xi(A):=0$ for $A \in \mathcal{M}_{1}^{\beta, \eta}$. Remark 5.3 in Okada (2019) verified that $\Xi$ is well defined. Let $g:=\left|\operatorname{Im} \sigma_{1}\right|, h:=\left|\operatorname{Im} \sigma_{2}\right|$ and $A(x):=x E-A$ for $x \in \mathbb{R}$ and a matrix $A$, where $E$ is an identity of appropriate size. Recall that $\lambda(A)$ is the maximum eigenvalue of $A$. Let $A_{r_{0}}^{(j)}:=\left(a_{i, l}\right)_{1 \leq i, l \leq j}$ if $a_{i, i}=1$ and $a_{i, l}=r_{0}$ for any $1 \leq i \neq l \leq j$.

Proposition 2.2. For any $j \geq 2, A \in \Xi^{-1}(\{(j-1)(1-\tilde{r})\}) \cap \mathcal{M}_{j}^{\beta, \eta}$ with $A=$ $B^{\sigma_{1}} \boxplus_{r} C^{\sigma_{2}}, 0<r \leq \tilde{r}<1$ and $x>\lambda\left(A_{\tilde{r}}^{(j)}\right)$,

(i) $\operatorname{det}(B(x)), \operatorname{det}(C(x))>0$,

(ii) there exist $y_{1}(A(x)), \ldots, y_{j}(A(x))$ and $y_{i}(A(x))>0$ for any $1 \leq i \leq j$,

(iii) $\lambda(A) \leq \lambda\left(A_{\tilde{r}}^{(j)}\right)=1+(j-1) \tilde{r}$,

$(i v) 0 \leq \chi(A(x)) \leq \chi\left(A_{\tilde{r}}^{(j)}(x)\right)$.

Proof: Without loss of generality, we assume that $\sigma_{1}(i)=i$ for $1 \leq i \leq g$ and $\sigma_{2}(i)=i+g$ for $1 \leq i \leq h$. We pick $r_{1}$ and $r_{2}$ such that $0<r \leq \tilde{r} \leq r_{1}, r_{2}<1$, 
$(j-1) \tilde{r}=(g-1) r_{2}+(h-1) r_{1}+r, B \in \Xi^{-1}\left(\left\{(g-1)\left(1-r_{2}\right)\right\}\right)$ and $C \in \Xi^{-1}(\{(h-$ $\left.\left.1)\left(1-r_{1}\right)\right\}\right)$. Given that $(i i)$ yields that $A(x)$ is a regular matrix, we obtain (iii) by (ii). Then, we only show $(i)$, (ii) and $(i v)$ by induction on $j$. For $j=2$, it is trivial that $(i)$, $(i i)$ and $(i v)$ hold. For $j \in \mathbb{N} \cap\{1,2\}^{c}$, we assume that the claim holds for $2, \ldots, j-1$. First, we show $(i)$. Given that $\lambda\left(A_{r_{2}}^{(g)}\right)=1+(g-1) r_{2}, \lambda\left(A_{r_{1}}^{(h)}\right)=$ $1+(h-1) r_{1}$ and $\lambda\left(A_{\tilde{r}}^{(j)}\right)=1+(j-1) \tilde{r}$, we have $\lambda\left(A_{r_{2}}^{(g)}\right) \vee \lambda\left(A_{r_{1}}^{(h)}\right)<\lambda\left(A_{\tilde{r}}^{(j)}\right)$. Thus, assuming $($ iii $)$ results in $\operatorname{det}(B(x))>0$ under $x>\lambda\left(A_{r_{2}}^{(g)}\right)$ and $\operatorname{det}(C(x))>0$ under $x>\lambda\left(A_{r_{1}}^{(h)}\right)$. Therefore, we obtain $(i)$.

Next, we show $(i i)$. First, we prove the following:

$$
1-r^{2} \chi(B(x)) \chi(C(x))>0 \text {. }
$$

The simple computation yields the following:

$$
\chi\left(A_{r_{2}}^{(g)}(x)\right)=\frac{g}{x-(g-1) r_{2}-1}, \quad \chi\left(A_{r_{1}}^{(h)}(x)\right)=\frac{h}{x-(h-1) r_{1}-1}
$$

and hence for $x>\lambda\left(A_{\tilde{r}}^{(j)}\right)$

$$
\begin{aligned}
1-r^{2} \chi\left(A_{r_{2}}^{(g)}(x)\right) \chi\left(A_{r_{1}}^{(h)}(x)\right) & =\frac{\left(x-(g-1) r_{2}-1\right)\left(x-(h-1) r_{1}-1\right)-r^{2} g h}{\left(x-(g-1) r_{2}-1\right)\left(x-(h-1) r_{1}-1\right)} \\
& >\frac{\left((h-1) r_{1}+r\right)\left((g-1) r_{2}+r\right)-r^{2} g h}{\left(x-(g-1) r_{2}-1\right)\left(x-(h-1) r_{1}-1\right)} \\
& \geq \frac{((h-1) r+r)((g-1) r+r)-r^{2} g h}{\left(x-(g-1) r_{2}-1\right)\left(x-(h-1) r_{1}-1\right)}=0 .
\end{aligned}
$$

Then, given that $\lambda\left(A_{\tilde{r}}^{(j)}\right) \geq \lambda\left(A_{r_{2}}^{(g)}\right) \vee \lambda\left(A_{r_{1}}^{(h)}\right)$, the assumption of $(i v)$ yields that for $x>\lambda\left(A_{\tilde{r}}^{(j)}\right)$

$$
0 \leq \chi(B(x)) \leq \chi\left(A_{r_{2}}^{(g)}(x)\right), \quad 0 \leq \chi(C(x)) \leq \chi\left(A_{r_{1}}^{(h)}(x)\right)
$$

and

$$
1-r^{2} \chi(B(x)) \chi(C(x)) \geq 1-r^{2} \chi\left(A_{r_{2}}^{(g)}(x)\right) \chi\left(A_{r_{1}}^{(h)}(x)\right)>0 .
$$

Then, we obtain (2.3). In addition, the assumption of $\left(\right.$ ii) results in $x>\lambda\left(A_{\tilde{r}}^{(j)}\right)>$ $\lambda\left(A_{\tilde{r}}^{(g)}\right) \vee \lambda\left(A_{\tilde{r}}^{(h)}\right), y_{i}(B(x))>0$ for $1 \leq i \leq g$ and $y_{i}(C(x))>0$ for $1 \leq i \leq h$. By the same argument as that of the proof of Proposition 5.2 in Okada (2019), (2.3) and the assumption of $(i i)$, we find the existence of $y_{1}(A(x)), \ldots, y_{j}(A(x))$,

$$
\begin{aligned}
& \left(y_{1}(A(x)), \ldots, y_{g}(A(x))\right)=\left(1+r \sum_{i=g+1}^{j} y_{i}(A(x))\right)\left(y_{1}(B(x)), \ldots, y_{g}(B(x))\right), \\
& \left(y_{g+1}(A(x)), \ldots, y_{j}(A(x))\right)=\left(1+r \sum_{i=1}^{g} y_{i}(A(x))\right)\left(y_{1}(C(x)), \ldots, y_{h}(C(x))\right)
\end{aligned}
$$

and

$$
\begin{aligned}
\sum_{i=1}^{g} y_{i}(A(x)) & =\frac{\sum_{i=1}^{g} y_{i}(B(x))+r \sum_{i=1}^{g} y_{i}(B(x)) \sum_{i=1}^{h} y_{i}(C(x))}{1-r^{2} \sum_{i=1}^{g} y_{i}(B(x)) \sum_{i=1}^{h} y_{i}(C(x))}>0 \\
\sum_{i=g+1}^{j} y_{i}(A(x)) & =\frac{\sum_{i=1}^{h} y_{i}(C(x))+r \sum_{i=1}^{g} y_{i}(B(x)) \sum_{i=1}^{h} y_{i}(C(x))}{1-r^{2} \sum_{i=1}^{g} y_{i}(B(x)) \sum_{i=1}^{h} y_{i}(C(x))}>0 .
\end{aligned}
$$


Hence, we have the desired result.

Finally, we show (iv). For $s, a, b \geq 0$ with $1-s^{2} a b>0$, set

$$
f(s, a, b):=\frac{a+b+2 s a b}{1-s^{2} a b} .
$$

Since (ii) yields that $A(x), B(x)$ and $C(x)$ are regular matrices, by the Schur complement, we have

$$
\begin{aligned}
& \left(B^{\sigma_{1}} \boxplus_{r} C^{\sigma_{2}}\right)(x)^{-1} \\
& =\left(\begin{array}{cc}
\left(B(x)-R(C(x))^{-1} R^{T}\right)^{-1} & -\left(B(x)-R(C(x))^{-1} R^{T}\right)^{-1} R(C(x))^{-1} \\
-(C(x))^{-1} R^{T}\left(B(x)-R(C(x))^{-1} R^{T}\right)^{-1} & \left(C(x)-R^{T}(B(x))^{-1} R\right)^{-1}
\end{array}\right)
\end{aligned}
$$

where $R$ is the $g \times h$-matrix all of whose entries are equal to $-r$. Hence, by the simple computation, each summation of all the entries are given as follows:

$$
\begin{aligned}
& \frac{\chi(B(x))}{1-r^{2} \chi(B(x)) \chi(C(x))}, \quad \frac{r \chi(B(x)) \chi(C(x))}{1-r^{2} \chi(B(x)) \chi(C(x))}, \\
& \frac{r \chi(B(x)) \chi(C(x))}{1-r^{2} \chi(B(x)) \chi(C(x))} \text { and } \frac{\chi(C(x))}{1-r^{2} \chi(B(x)) \chi(C(x))}
\end{aligned}
$$

given that $1-r^{2} \chi(B(x)) \chi(C(x))>0$. Since $\operatorname{det}(B(x))$, $\operatorname{det}(C(x)) \geq 0$, we have

$$
\chi\left(\left(B^{\sigma_{1}} \boxplus_{r} C^{\sigma_{2}}\right)(x)\right)=f(r, \chi(B(x)), \chi(C(x)))
$$

(see another proof in Proposition 5.2 in Okada, 2019). Then, by the assumption of (iv), we obtain

$$
0 \leq f(r, \chi(B(x)), \chi(C(x))) \leq f\left(r, \chi\left(A_{r_{2}}^{(g)}(x)\right), \chi\left(A_{r_{1}}^{(h)}(x)\right)\right)
$$

since $f(s, a, b)$ monotonically increases in $a$ and $b$ (see the proof of Lemma 5.2 in Okada, 2019). Hence, it suffices to show

$$
f\left(r, \chi\left(A_{r_{2}}^{(g)}(x)\right), \chi\left(A_{r_{1}}^{(h)}(x)\right)\right) \leq f\left(\tilde{r}, \chi\left(A_{\tilde{r}}^{(g)}(x)\right), \chi\left(A_{\tilde{r}}^{(h)}(x)\right)\right) .
$$

First, we show that if the maximum of $f\left(r, \chi\left(A_{r_{2}}^{(g)}(x)\right), \chi\left(A_{r_{1}}^{(h)}(x)\right)\right)$ is attained at $r=r_{1}$ fixing the value $(h-1) r_{1}+r$ and $(g-1) r_{2}$ if $r_{1} \leq r_{2}$ or $g=1$. If we set $p=x-(g-1) r_{2}-1$ and $q=x-(h-1) r_{1}-r-1$,

$$
f\left(r, \chi\left(A_{r_{2}}^{(g)}(x)\right), \chi\left(A_{r_{1}}^{(h)}(x)\right)\right)=\frac{p h+g(q+r)+2 r g h}{p(q+r)-r^{2} g h}:=\hat{f}(r) .
$$

In addition, if $r_{1} \leq r_{2},(-q-1+x) / h \leq r_{2}$ holds. Hence, it suffices to show the maximum of $\hat{f}(r)$ for $0 \leq r \leq(-q-1+x) / h$ is attained at $r=(-q-1+x) / h$. Moreover, fixing the values $p$ and $q$, we obtain

$$
\frac{\partial \hat{f}(r)}{\partial r}=\frac{g^{2} h(2 h+1)\left(r+\frac{g q+h p}{g+2 g h}\right)^{2}-\frac{h(g q+h p)^{2}}{1+2 h}+2 q p g h-h p^{2}}{\left(p(q+r)-r^{2} g h\right)^{2}} .
$$

Note that $p, q \geq 0$ for $x \geq(j-1) \tilde{r}+1$ and hence $-(g q+h p) /(g+2 g h) \leq 0$. Thus, the maximum of $\hat{f}(r)$ is attained at $r=(-q-1+x) / h$ or 0 . Then, by an elementary computation, we have that $\hat{f}((-q-1+x) / h) \geq \hat{f}(0)$ for $x>(j-1) \tilde{r}+1$. Therefore, given that the maximum of $\hat{f}(r)$ is attained at $r=(-q-1+x) / h$, the maximum of $\hat{f}(r)$ is attained at $r=r_{1}$.

Now, we show (2.5). By the symmetry of $r_{1}$ and $r_{2}$, it suffices to show (2.5) if $r_{1} \leq r_{2}$ and $g \wedge h \geq 2$. In addition, since the maximum of $\hat{f}(r)$ is attained at $r=r_{1}$ 
fixing the value $(h-1) r_{1}+r$ and $(g-1) r_{2}$, it suffices to show (2.5) if $r=r_{1}$ and $r_{1} \leq r_{2}$. Note that

$$
f\left(r, \chi\left(A_{r_{2}}^{(g)}(x)\right), \chi\left(A_{r}^{(h)}(x)\right)\right)=f\left(r, \chi\left(A_{r_{2}}^{(g)} \boxplus_{r} A^{(1)}(x)\right), \chi\left(A_{r}^{(h-1)}(x)\right)\right) .
$$

Set $r_{i+1}:=\left((g+i-3) r_{i}+r\right) /(g+i-2)$ for $2 \leq i \leq h+1$. With the aid of the result for $g \wedge h=1$, we obtain

$$
f\left(r, \chi\left(A_{r_{2}}^{(g)}(x)\right), \chi\left(A^{(1)}(x)\right)\right) \leq f\left(r_{3}, \chi\left(A_{r_{3}}^{(g)}(x)\right), \chi\left(A^{(1)}(x)\right)\right)=\chi\left(A_{r_{3}}^{(g+1)}(x)\right) .
$$

Since $f(s, a, b)$ is increasing on $a$, we have

$$
f\left(r, \chi\left(A_{r_{2}}^{(g)}(x)\right), \chi\left(A_{r}^{(h)}(x)\right)\right) \leq f\left(r, \chi\left(A_{r_{3}}^{(g+1)}(x)\right), \chi\left(A_{r}^{(h-1)}(x)\right)\right) .
$$

If $h \geq 3$, we inductively obtain

$$
f\left(r, \chi\left(A_{r_{i}}^{(g+i-2)}(x)\right), \chi\left(A_{r}^{(h-i+2)}(x)\right)\right) \leq f\left(r, \chi\left(A_{r_{i+1}}^{(g+i-1)}(x)\right), \chi\left(A_{r}^{(h-i+1)}(x)\right)\right) .
$$

Hence, we have

$$
\begin{aligned}
f\left(r, \chi\left(A_{r_{2}}^{(g)}(x)\right), \chi\left(A_{r}^{(h)}(x)\right)\right) & \leq f\left(r, \chi\left(A_{r_{h+1}}^{(g+h-1)}(x)\right), \chi\left(A^{(1)}(x)\right)\right) \\
& \leq f\left(r_{h+2}, \chi\left(A_{r_{h+2}}^{(g+h-1)}(x)\right), \chi\left(A^{(1)}(x)\right)\right) .
\end{aligned}
$$

Thus, given that $r_{h+2}=\tilde{r}$, we obtain (2.5) if $r=r_{1}$ and $r_{1} \leq r_{2}$. Therefore, we obtain (2.5) and hence (iv).

To state the following propositions, we provide some definitions concerning the configuration of points in $\mathbb{Z}^{2}$. Given real valued $j \times j$-matrices $M:=\left(m_{i, l}\right)_{1 \leq i, l \leq j}$, $M^{\prime}:=\left(m_{i, l}^{\prime}\right)_{1 \leq i, l \leq j}$, let

$$
\begin{aligned}
\mathcal{E}\left[M, M^{\prime}\right] & =\mathcal{E}\left[M, M^{\prime}\right](j, n) \\
& :=\left\{\vec{x} \in\left(\mathbb{Z}^{2}\right)^{j}: m_{i, l} \leq d\left(x_{i}, x_{l}\right) \leq m_{i, l}^{\prime} \text { for any } 1 \leq i \neq l \leq j\right\} .
\end{aligned}
$$

Note that the set is independent of diagonal elements of a matrix. When for $1 \leq i \neq$ $l \leq j, m_{i, l}$ is constant and $m_{i, l}^{\prime}$ be, we simply write $\mathcal{E}\left[\left(m_{i, l}\right),\left(m_{i, l}^{\prime}\right)\right]$. For $A \in \mathcal{M}_{j}^{\beta, \eta}$, $\delta^{\prime}>0$ let

$$
\hat{\mathcal{E}}_{\delta^{\prime}}[A]=\hat{\mathcal{E}}_{\delta^{\prime}}[A](j, n):=\mathcal{E}\left[\left(\frac{1}{2^{j}} n^{1-a_{i, l}}\right)_{1 \leq i, l \leq j},\left(2^{j} n^{1-a_{i, l}+\delta^{\prime}}\right)_{1 \leq i, l \leq j}\right] .
$$

Now, we provide the following proposition. The proof is given in Proposition 4.1 in Okada (2019).

Proposition 2.3. For any $\delta^{\prime}>0$ and $0<\eta \leq(1-\beta) \wedge \beta$ there exists $n_{0} \in \mathbb{N}$ such that for any $n \geq n_{0}$ and $\vec{x} \in \mathcal{E}\left[\left(n^{\eta}\right),\left(n^{\beta}\right)\right]$ there exists $A \in \mathcal{M}_{j}^{\beta, \eta}$ such that $\vec{x} \in \hat{\mathcal{E}}_{\delta^{\prime}}[A]$ holds.

Next, we select $\delta_{0}>0$. As per Proposition 2.3, for $\delta^{\prime}>0, \vec{x} \in \mathcal{E}\left[\left(n^{\eta}\right),\left(n^{\beta}\right)\right]$ and all sufficiently large $n \in \mathbb{N}$, we can set

$$
g_{\delta^{\prime}}(\vec{x}):=\inf \left\{j \lambda(B)^{-1}: \vec{x} \in \hat{\mathcal{E}}_{\delta^{\prime}}[B], B \in \mathcal{M}_{j}^{\beta, \eta}\right\} .
$$

Next, we provide the following key proposition.

Proposition 2.4. For any $\epsilon>0$ there exists $C>0$ such that for any $n \in \mathbb{N}$ and $0<\delta^{\prime}<\delta_{0}$

$$
\sum_{\vec{x} \in \mathcal{E}\left[\left(n^{\eta}\right),\left(n^{\beta}\right)\right]} n^{-2 \alpha g_{\delta^{\prime}}(\vec{x})} \leq C n^{\hat{\rho}_{j}(\alpha, \beta)+\epsilon} .
$$


Proof: (iii) in Proposition 2.2 implies

$$
\min _{A \in \Xi^{-1}(\{t\})} j \lambda\left(\left(a_{i, l}\right)_{1 \leq i, l \leq j}\right)^{-1}=\frac{j}{j-t} .
$$

Hence, we obtain

$$
\min _{A \in \Xi^{-1}(\{t\})} g_{\delta^{\prime}}(\vec{x})=\frac{j}{j-t} .
$$

Thus, applying the same argument as the proof of Proposition 5.4 in Okada (2019) yields the following result: for any $\delta^{\prime}>0$ there exists $C>0$ such that for any $n \geq n_{0}$, the left-hand side in (2.6) is bounded by

$$
\begin{aligned}
& C \max _{0 \leq t \leq(j-1) \beta} \max _{A \in \Xi^{-1}(\{t\})} \sum_{\vec{x} \in \hat{\mathcal{E}}_{\delta^{\prime}}[A]} n^{-2 \alpha g_{\delta^{\prime}}(\vec{x})} \\
\leq C & \max _{0 \leq t \leq(j-1) \beta} n^{2 t+2+\epsilon / 2} \max _{A \in \Xi^{-1}(\{t\})} n^{-2 \alpha g_{\delta^{\prime}}(\vec{x})} \leq C \max _{0 \leq t \leq(j-1) \beta} n^{2 t+2+\epsilon} n^{-2 \alpha j /(j-t) .}
\end{aligned}
$$

Therefore, given that

$$
\max _{0 \leq t \leq(j-1) \beta} 2 t+2-\frac{2 \alpha j}{j-t}=\hat{\rho}_{j}(\alpha, \beta),
$$

we obtain the desired result.

Finally, we give the following proposition and remark. We use them to show Proposition 2.10 in the next section. Let \|\| be the matrix norm.

Proposition 2.5. For any $\epsilon>0$, there exists $\delta>0$ such that for any $A \in \mathcal{M}_{j}^{\beta, \eta}$, symmetric matrix $\tilde{A}$ with $\|A-\tilde{A}\| \leq \delta, \tilde{A}$ is a regular matrix and

$$
\left|\lambda(A)^{-1}-\lambda(\tilde{A})^{-1}\right| \leq \epsilon .
$$

Remark 2.6. It is trivial that Proposition 2.5 yields the following. For any $\epsilon>0$, there exists $\delta>0$ such that for any $n \in \mathbb{N}$ and $A \in \mathcal{M}_{j}^{\beta, \eta}$, symmetric matrix $\tilde{A}$ with $\|n A-\tilde{A}\| \leq \delta n$,

$$
\left|\frac{\lambda(A)^{-1}}{n}-\lambda(\tilde{A})^{-1}\right| \leq \frac{\epsilon}{n} .
$$

Proof of Proposition 2.5: Since we showed the first claim in Proposition 5.5 in Okada (2019), we now prove the second claim. Let

$$
\begin{aligned}
\hat{\mathcal{M}}_{j}^{\beta, \eta}:=\left\{\left(a_{i, l}\right)_{1 \leq i, l \leq j}: \text { symmetric, } 1-\eta \leq a_{i, i} \leq 1+\eta,\right. \\
\left.1-\beta-\eta \leq a_{i, l} \leq 1-\eta / 2 \text { for any } 1 \leq i \neq l \leq j\right\} .
\end{aligned}
$$

Since roots of polynomial are continuous as for polynomial coefficients, $\lambda: \hat{\mathcal{M}}_{j}^{\beta, \eta} \rightarrow$ $\mathbb{R}$ is continuous. Thus, by the compactness of $\hat{\mathcal{M}}_{j}^{\beta, \eta}$ (see a similar proof in Proposition 5.5 in Okada, 2019), it suffices to show

$$
\inf _{A \in \hat{\mathcal{M}}_{j}^{\beta, \eta}} \lambda(A)>0 .
$$

Note that all the entries of $A \in \hat{\mathcal{M}}_{j}^{\beta, \eta}$ are positive. Then, the application of the Perron-Frobenius Theorem yields the following: $\lambda(A)>0$ for any $A \in \hat{\mathcal{M}}_{j}^{\beta, \eta}$ 
(see Reed and Simon, 1978). Hence, by the compactness of $\hat{\mathcal{M}}_{j}^{\beta, \eta}$, we have (2.7). Therefore, we obtain the desired result.

To state the following lemmas, we let the eigenvalues of a symmetric matrix $A$ be $\lambda(A)=\lambda_{1}(A) \geq \lambda_{2}(A) \geq \ldots \geq \lambda_{j}(A), \phi_{i}=\left(\phi_{i, 1}, \ldots, \phi_{i, j}\right)$ the corresponding orthonormal eigenvectors to $\lambda_{i}(A)$ and $h_{i}(A):=\sum_{l=1}^{j} \phi_{i, l} \phi_{i, 1}$.

Lemma 2.7. There exist $\delta^{\prime}>0$ and $\delta>0$ such that if $\left\|A_{1-b}^{(j)}-\tilde{A}\right\| \leq \delta n$ hold for $\eta \leq b \leq \beta+\eta, n \in \mathbb{N}$ and symmetric matrix $\tilde{A}$, then

$$
\left|\lambda(\tilde{A})^{-1}-\lambda_{2}(\tilde{A})^{-1}\right| \geq \frac{\delta^{\prime}}{n} .
$$

Lemma 2.8. There exist $\delta^{\prime}>0$ and $\delta>0$ such that if $\left\|n A_{1-b}^{(j)}-\tilde{A}\right\| \leq \delta n$ hold for $\eta \leq b \leq \beta+\eta, n \in \mathbb{N}$ and symmetric matrix $\tilde{A}$, then

$$
h_{1}(\tilde{A}) \geq \delta^{\prime} .
$$

Proof of Lemma 2.7: (2.7) makes it sufficient to show that there exist $\delta^{\prime}>0$ and $\delta>0$ such that if $\left\|n A_{1-b}^{(j)}-\tilde{A}\right\| \leq \delta n$ holds for $n \in \mathbb{N}$, then

$$
\left|\lambda(\tilde{A})-\lambda_{2}(\tilde{A})\right| \geq \delta^{\prime} n \text {. }
$$

Note that the simple computation yields

$$
\begin{aligned}
& \lambda\left(A_{1-b}^{(j)}\right)=1+(j-1)(1-b), \\
& \lambda_{2}\left(A_{1-b}^{(j)}\right)=b .
\end{aligned}
$$

Given that the roots of polynomials are continuous for polynomial coefficients, we obtain the desired result.

Proof of Lemma 2.8: Using the Perron-Frobenius Theorem, we have the following: $\phi_{1, i}>0$ for any $1 \leq i \leq j$ and $A \in \hat{\mathcal{M}}_{j}^{\beta, \eta}$. Thus, there exists $c>0$ such that $\phi_{1, i} \geq c$ for any $1 \leq i \leq j$ and $A \in \hat{\mathcal{M}}_{j}^{\beta, \eta}$, which satisfies the assumption, since $\lambda\left(A_{1-b}^{(j)}\right)=1+(j-1)(1-b)$. Therefore, we obtain the desired result.

2.3. Proof of Theorem 1.1. In this section, we prove Theorem 1.1 using the results from the previous section. To show Theorem 1.1, we first prepare the following lemma and proposition.

Lemma 2.9. Fix $x_{1}, \ldots, x_{j} \in D(0, n)$. It holds that

$$
\begin{aligned}
& P^{x_{1}}\left(K\left(\tau_{n},\left\{x_{1}, \ldots, x_{j}\right\}\right)>u\right)=\sum_{i=1}^{j} h_{i}\left(\frac{\lambda_{i}-1}{\lambda_{i}}\right)^{u} \\
& \text { for } u=0,1, \ldots, \text { where } \lambda_{i}=\lambda_{i}\left(\left(G_{n}\left(x_{v}, x_{l}\right)\right)_{1 \leq v, l \leq j}\right) \text { and } h_{i} \quad:= \\
& h_{i}\left(\left(G_{n}\left(x_{v}, x_{l}\right)\right)_{1 \leq v, l \leq j}\right) .
\end{aligned}
$$

Proof: Note that for any $m \in \mathbb{N}$

$$
\begin{aligned}
E^{x_{1}}\left[K\left(\tau_{n},\left\{x_{1}, \ldots, x_{j}\right\}\right)^{m}\right] & =E^{x_{1}}\left[\left(\sum_{l=0}^{\tau_{n}} 1_{\left\{S_{l} \in\left\{x_{1}, \ldots, x_{j}\right\}\right\}}\right)^{m}\right] \\
& =\sum_{i_{1}, \ldots, i_{m}=0}^{\infty} E^{x_{1}}\left[\prod_{b=1}^{m} 1_{\left\{S_{i_{b}} \in\left\{x_{1}, \ldots, x_{j}\right\}, S_{i_{b-1}}, \ldots, S_{i_{b}} \notin \partial D(0, n)\right\}}\right],
\end{aligned}
$$


where $i_{-1}=0$. By the Markov property, it equals to

$$
\sum_{k=1}^{m} \sum_{\substack{c_{1}, \ldots, c_{k} \in[1, m] \\
c_{1}+\ldots+c_{k}=m}}\left(\begin{array}{c}
m \\
c_{1}, \ldots, c_{k}
\end{array}\right) \sum_{\substack{z_{i} \in\left\{x_{1}, \ldots, x_{j}\right\} \\
1 \leq \forall i \leq k}} \sum_{0 \leq u_{1}<\ldots<u_{k}<\infty} \prod_{b=1}^{k} q_{u_{b}-u_{b-1}}\left(z_{b-1}, z_{b}\right),
$$

where $q_{u}\left(z, z^{\prime}\right)=E^{z}\left[1_{\left\{S_{u}=z^{\prime}, u<\tau_{n}\right\}}\right], z_{0}=x_{1}$ and $u_{0}=0$. Given that the remaining proof is the same as that of Csáki et al. (2005), we omit it.

Proposition 2.10. For any $\epsilon>0$ there exist $C, c>0$ and $\delta^{\prime}>0$ such that for any $n \in \mathbb{N}$ and uniformly in $\vec{x} \in \hat{\mathcal{E}}_{\delta^{\prime}}[A]$ with $x_{1}, \ldots, x_{j} \in D(0, n)$,

$$
P\left(\vec{x} \in \mathcal{R}_{j, n}(\alpha)\right) \leq C n^{-2 \alpha g_{\delta^{\prime}}(\vec{x})+\epsilon}
$$

and uniformly in $\vec{x} \in \hat{\mathcal{E}}_{\delta^{\prime}}\left[A_{1-b}^{(j)}\right]$ with $x_{1}, \ldots, x_{j} \in D(0, n / 10)$ and $\eta \leq b \leq \beta+\eta$,

$$
c n^{-2 \alpha g_{\delta^{\prime}}(\vec{x})-\epsilon} \leq P\left(\vec{x} \in \mathcal{R}_{j, n}(\alpha)\right)
$$

Proof: We fix $\epsilon>0$. First, we show the upper bound. Lemma 2.9 yields

$$
\begin{aligned}
& P\left(K\left(\tau_{n},\left\{x_{1}, \ldots, x_{j}\right\}\right) \geq u, T_{x_{1}}=\min _{1 \leq i \leq j} T_{x_{i}}\right) \\
\leq & P\left(K\left(\tau_{3 n},\left\{x_{1}, \ldots, x_{j}\right\}\right) \geq u, T_{x_{1}}=\min _{1 \leq i \leq j} T_{x_{i}}\right) \\
\leq & \sum_{k=1}^{j}\left|h_{k}\right|\left(1-\lambda_{k}\left(\left(G_{3 n}\left(x_{i}, x_{l}\right)\right)_{1 \leq i, l \leq j}\right)^{-1}\right)^{u-1} .
\end{aligned}
$$

Since $\max _{1 \leq i, l \leq j}\left|\phi_{i, l}\right| \leq 1$, it holds that $\max _{1 \leq i \leq j}\left|h_{i}\right| \leq j$. In addition, by (2.2) to achieve uniformity in $\vec{x} \in \hat{\mathcal{E}}_{\delta^{\prime}}[A]$ with $x_{1}, \ldots, x_{j} \in D(0, n)$,

$$
\begin{aligned}
\left|G_{3 n}\left(x_{i}, x_{l}\right)-a_{i, l} \frac{2 \log n}{\pi}\right| & \leq\left|\frac{2}{\pi}\left(\log n-\log d\left(x_{i}, x_{l}\right)^{+}+o(1)\right)-a_{i, l} \frac{2 \log n}{\pi}\right| \\
& \leq \max \frac{2 \log n}{\pi}\left(\left|b_{i, l}-a_{i, l}\right|+\delta^{\prime}\right) \\
& =\left(o(1)+o\left(\delta^{\prime}\right)\right) \log n,
\end{aligned}
$$

where the above-mentioned maximum is over $b_{i, l}=a_{i, l}+o(1)$ with $1 \leq i, l \leq j$ and $o\left(\delta^{\prime}\right)$ means that it goes to 0 when $\delta^{\prime}$ converges to 0 . Therefore, by Proposition 2.5 and the symmetry of $x_{1}, \ldots, x_{j}$,

$$
P\left(K\left(\tau_{n},\left\{x_{1}, \ldots, x_{j}\right\}\right) \geq u\right) \leq C n^{-2 \alpha g_{\delta^{\prime}}(\vec{x})+\epsilon}
$$

if we select $u=\left\lceil 4 \alpha j(\log n)^{2} / \pi\right\rceil$ and hence the desired upper bound is completed.

Next, we show the lower bound. By (2.1), there exists $c>0$ such that for any $n \in \mathbb{N}$ and $x_{1} \in D(0, n / 10)$

$$
P\left(T_{x_{1}}<\tau_{n}\right) \geq c .
$$


Again, Lemma 2.9 provides that for $\vec{x} \in \hat{\mathcal{E}}_{\delta^{\prime}}\left[A_{1-b}^{(j)}\right]$ with $x_{1}, \ldots, x_{j} \in D(0, n / 10)$ and $\eta \leq b \leq \beta+\eta$,

$$
\begin{aligned}
& P\left(K\left(\tau_{n},\left\{x_{1}, \ldots, x_{j}\right\}\right) \geq u\right) \\
\geq & P\left(T_{x_{1}}<\tau_{n}\right) P^{x_{1}}\left(K\left(\tau_{n},\left\{x_{1}, \ldots, x_{j}\right\}\right) \geq u\right) \\
\geq & c\left(h_{1}\left(1-\lambda\left(\left(G_{n}\left(x_{i}, x_{l}\right)\right)_{1 \leq i, l \leq j}\right)^{-1}\right)^{u}-\sum_{k=2}^{j}\left|h_{k}\right|\left(1-\lambda_{k}\left(\left(G_{n}\left(x_{i}, x_{l}\right)\right)_{1 \leq i, l \leq j}\right)^{-1}\right)^{u}\right) .
\end{aligned}
$$

Then, if we select $u=\left\lceil 4 \alpha j(\log n)^{2} / \pi\right\rceil$, with the aid of (2.8), Proposition 2.5 and Lemmas 2.7 and 2.8 indicates that the most right-hand side in (2.9) is larger than

$$
\begin{aligned}
& c\left(1-\lambda\left(\left(G_{n}\left(x_{i}, x_{l}\right)\right)_{1 \leq i, l \leq j}\right)^{-1}\right)^{\left\lceil 4 \alpha j(\log n)^{2} / \pi\right\rceil} \\
\geq & \exp \left(-2 \alpha j \lambda\left(\left(\frac{\pi G_{n}\left(x_{i}, x_{l}\right)}{2 \log n}\right)_{1 \leq i, l \leq j}\right)^{-1} \log n+o(\log n)\right) \\
\geq & c n^{-2 \alpha g_{\delta^{\prime}}(\vec{x})-\epsilon}
\end{aligned}
$$

for $x_{1}, \ldots, x_{j} \in D(0, n / 10)$. Therefore, we obtain the desired lower bound.

Proof of the upper bound in Theorem 1.1: Propositions 2.4 and 2.10 yield that for any $\epsilon>0$, there exists $C>0$ such that for any $n \in \mathbb{N}$,

$$
\sum_{\vec{x} \in \mathcal{E}\left[\left(n^{\eta}\right),\left(n^{\beta}\right)\right]} P\left(\vec{x} \in \mathcal{R}_{j, n}(\alpha)\right) \leq C n^{\hat{\rho}_{j}(\alpha, \beta)+\epsilon} .
$$

Hence, we need only extend the result for " $\mathcal{E}\left[\left(n^{\eta}\right),\left(n^{\beta}\right)\right]$ " to " $\mathcal{E}\left[(0),\left(n^{\beta}\right)\right]$ ". This proof is the same as that of the upper bound in Theorem 2.1 in Okada (2019), and hence we have omitted it.

Proof of the lower bound in Theorem 1.1: It is trivial that $j \lambda\left(A_{1-b}^{(j)}\right)^{-1}=j /(1+$ $(j-1)(1-b))$. Therefore, if we consider $\vec{x} \in \mathcal{E}\left[\left(n^{b}\right),\left(5 j n^{b}\right)\right]$ with $x_{1}, \ldots, x_{j} \in$ $D(0, n / 10)$ for $\eta<b<\beta+\eta$, then applying Proposition 2.10 yields the following result:

$$
P\left(\vec{x} \in \mathcal{R}_{j, n}(\alpha)\right) \geq \exp \left(-\frac{2 j \alpha \log n}{1+(j-1)(1-b)}+o(\log n)\right) .
$$

The proof is omitted as it is the same as that of the lower bound in Theorem 2.1 in Okada (2019).

\section{References}

M. J. A. M. Brummelhuis and H. J. Hilhorst. Covering of a finite lattice by a random walk. Phys. A 176 (3), 387-408 (1991). MR1130067.

E. Csáki, A. Földes, P. Révész, J. Rosen and Z. Shi. Frequently visited sets for random walks. Stochastic Process. Appl. 115 (9), 1503-1517 (2005). MR2158017.

O. Daviaud. Extremes of the discrete two-dimensional Gaussian free field. Ann. Probab. 34 (3), 962-986 (2006). MR2243875.

C. Dellacherie, S. Martinez and J. San Martin. Inverse M-matrices and ultrametric matrices, volume 2118 of Lecture Notes in Mathematics. Springer, Cham (2014). ISBN 978-3-319-10297-9; 978-3-319-10298-6. MR3289211. 
A. Dembo. Favorite points, cover times and fractals. In Lectures on probability theory and statistics, volume 1869 of Lecture Notes in Math., pages 1-101. Springer, Berlin (2005). MR2228383.

A. Dembo. Simple random covering disconnection, late and favorite points. In International Congress of Mathematicians. Vol. III, pages 535-558. Eur. Math. Soc., Zürich (2006). MR2275695.

A. Dembo, Y. Peres and J. Rosen. How large a disc is covered by a random walk in $n$ steps? Ann. Probab. 35 (2), 577-601 (2007). MR2308589.

A. Dembo, Y. Peres, J. Rosen and O. Zeitouni. Thick points for planar Brownian motion and the Erdös-Taylor conjecture on random walk. Acta Math. 186 (2), 239-270 (2001). MR1846031.

A. Dembo, Y. Peres, J. Rosen and O. Zeitouni. Cover times for Brownian motion and random walks in two dimensions. Ann. of Math. (2) 160 (2), 433-464 (2004). MR2123929.

A. Dembo, Y. Peres, J. Rosen and O. Zeitouni. Late points for random walks in two dimensions. Ann. Probab. 34 (1), 219-263 (2006). MR2206347.

P. Erdös and P. Révész. Problems and results on random walks. In Mathematical statistics and probability theory, Vol. B (Bad Tatzmannsdorf, 1986), pages 59-65. Reidel, Dordrecht (1987). MR922711.

G. F. Lawler. Intersections of random walks. Probability and its Applications. Birkhäuser Boston, Inc., Boston, MA (1991). ISBN 0-8176-3557-2. MR1117680.

S. Martínez, G. Michon and J. San Martín. Inverse of strictly ultrametric matrices are of Stieltjes type. SIAM J. Matrix Anal. Appl. 15 (1), 98-106 (1994). MR1257619.

I. Okada. Exponents for the number of pairs of nearly favorite points of simple random walk in $\digamma^{2}(2019+)$. To appear in Stoc. Process. Appl.

I. Okada. Geometric structures of late points of a two-dimensional simple random walk. Ann. Probab. 47 (5), 2869-2893 (2019). MR4021239.

M. Reed and B. Simon. Methods of modern mathematical physics. IV. Analysis of operators. Academic Press [Harcourt Brace Jovanovich, Publishers], New YorkLondon (1978). ISBN 0-12-585004-2. MR0493421.

J. Rosen. A random walk proof of the Erdös-Taylor conjecture. Period. Math. Hungar. 50 (1-2), 223-245 (2005). MR2162811. 